\title{
Path-dependent infinite-dimensional SDE with non-regular drift: an existence result
}

\author{
David Dereudre* \\ Sylvie Roelly ${ }^{\dagger}$
}

October 21, 2018

\begin{abstract}
We establish in this paper the existence of weak solutions of infinite-dimensional shift invariant stochastic differential equations driven by a Brownian term. The drift function is very general, in the sense that it is supposed to be neither bounded or continuous, nor Markov. On the initial law we only assume that it admits a finite specific entropy and a finite second moment. The originality of our method leads in the use of the specific entropy as a tightness tool and in the description of such infinitedimensional stochastic process as solution of a variational problem on the path space. Our result clearly improves previous ones obtained for free dynamics with bounded drift.
\end{abstract}

Key-words: Infinite-dimensional SDE, non-Markov drift, non-regular drift, variational principle, specific entropy.

\footnotetext{
*Laboratoire Paul Painlevé, UMR CNRS 8524, Université Lille1, 59655 Villeneuve d'Ascq Cedex, France, david.dereudre@univ-lille1.fr

${ }^{\dagger}$ Institut für Mathematik der Universität Potsdam, Am Neuen Palais 10, 14469 Potsdam, Germany, roelly@math.uni-potsdam.de
} 


\section{Introduction}

The main object of this paper is the infinite-dimensional stochastic differential equation (SDE)

$$
d X_{i}(t)=\mathbf{b}_{t}\left(\theta_{i} X\right) d t+d B_{i}(t), \quad i \in \mathbb{Z}^{d},
$$

on the configuration space $\Omega=\mathcal{C}([0, T], \mathbb{R})^{\mathbb{Z}^{d}}$, where the drift $\mathbf{b}:[0, T] \times \Omega$ is an adapted functional, $\theta_{i}$ denotes the space-shift on $\Omega$ by vector $-i$ and $\left(B_{i}\right)_{i \in \mathbb{Z}^{d}}$ is a sequence of independent real-valued Brownian motions.

Our aim is to prove the existence of a space-shift invariant weak solution of the SDE (1) on the finite time-interval $[0, T]$, where the drift $\mathbf{b}$ is supposed to be as general as possible, in particular non-Markov, non-regular and non bounded. Indeed, in Theorem 2.1, we solve the SDE (1) for a path-dependent drift which is local and admits a sublinear growth (see the precise assumptions in Section 2.3).

Let us illustrate our main result by a simple example. Let $\beta^{+} \neq \beta^{-}$be two functions defined on $\mathbb{R}^{\Delta}$ where $\Delta$ is a finite subset of $\mathbb{Z}^{d}$. Define first the function $b$ on $\mathbb{R}^{\mathbb{Z}^{d}}$ by

$$
b(x):=\beta^{+}\left(x_{\Delta}\right) \mathbb{1}_{\left\{x_{0} \geq \frac{1}{N} \sum_{i \in \Delta} x_{i}\right\}}+\beta^{-}\left(x_{\Delta}\right) \mathbb{1}_{\left\{x_{0}<\frac{1}{N} \sum_{i \in \Delta} x_{i}\right\}},
$$

where $x_{\Delta}:=\left(x_{i}\right)_{i \in \Delta}$ and $N$ is the cardinality of $\Delta$. It coincides with the function $\beta^{+}$(respectively $\beta^{-}$) if the 0 -coordinate $x_{0}$ is larger (respectively smaller) than the barycentre of $x_{\Delta}$. Introducing a $\delta$-delay (with $0<\delta<T$ ) consider now the drift $\mathbf{b}_{t}(\omega):=b(\omega(0 \vee(t-\delta))$. It leads to a stochastic differential delay equation (1) whose local discontinuous drift is uniformly sublinear (see (7)) as soon as the functions $\beta^{+}$and $\beta^{-}$admit a sublinear growth. In the above example the time memory of the drift is bounded by $\delta$, but our approach also allows to deal with path-dependent drift with long-term memory like $\mathbf{b}_{t}(\omega):=\int_{0}^{t} b(s, \omega(s)) d s$.

Note that SDE with non-Markov and non-regular drifts are relevant in many fields of applications like mathematical finance (see e.g. the stochastic functional differential equation with fixed or variable delay (2) and (3) satisfied by the stock price in [AHMP07]), biomathematics or physics, see e.g. [M97], or [TP01]. 
Let us briefly recall some results concerning weak solutions of infinitedimensional SDEs with additive noise.

In the very special Markovian case, when the drift only depends on the present time $\mathbf{b}_{t}(\omega)=\mathbf{b}_{t}(\omega(t))$, and the functions $x \mapsto \mathbf{b}_{t}(x)$ are regular and satisfy certain growth condition at infinity, weak solutions of (1) with values in a weighted $\ell^{2}$-space were constructed in [LR85] adapting a method used in [DR78]. For the existence of weak solutions of a Markov SDE with unbounded linear term the general theory of Dirichlet forms can also be used fruitfully, see e.g. [AR91].

If the drift is non-Markov but uniformly bounded, also up to the addition of an unbounded regular linear operator, imbedding the system of SDEs in an appropriate Hilbert space, it is possible to use Girsanov theory to provide a weak solution as element of this Hilbert space (see e.g. Chapter 10 in [DPZ92]).

For general non-Markov, non-regular and non bounded drifts $\mathbf{b}$, to our knowledge, till now there is no general weak existence result. The aim of this paper is to present a new entropy method to solve that question. Indeed, a fruitful approach to construct weak solutions of infinite systems of SDEs like (1) is to describe them as Gibbs measures on a path space. This point of view was initiated for gradient diffusions on a finite time interval in [D87] and developed later in [CRZ96]. The procedure includes two steps:

i) the construction of Gibbs measures on the path space associated to a suitable Hamiltonian $H$ depending on the drift $\mathbf{b}$ and on the initial Gibbsian law.

ii) the identification of (some of) them as weak solutions of (1).

When the uniform norm of the drift $\mathbf{b}$ is small enough, step $i$ ) can be done via the perturbative techniques of cluster expansion, as in [DPR06] and [RR14]. But recently a more general tool, first appeared in [GH96] and based on the compactness of the level sets of the specific entropy density, allowed to construct directly infinite-volume Gibbs measures associated to strong interaction [D09, DDG12]. This entropy method will be our first major tool. It will allow us to treat the case of a drift with sublinear growth, with an initial law which is not necessarily Gibbsian. Moreover the constructed solution inherits for free the finite entropy property of its approximations.

When the drift is Markov and Malliavin-differentiable, step ii) can be done via an integration by parts formula on the path space, as in [CRZ96]. In the 
general case, a variational principle which characterizes the shift invariant Gibbs measures as the minimizers of a so-called free energy functional, is more suitable. So, we will here identify the Gibbs measure solving a variational problem, as in [DPRZ02]: it will be our second major tool.

Our approach underlines to what extent tools from statistical mechanics can be powerful in the framework of stochastic analysis. Let us mention that this strategy has just been applied fruitfully in the framework of stochastic geometry to construct Gibbsian dynamics of tessellations by means of random cell divisions in continuous time, see [GST14]. There, the tightness tool is based on the level set of a space-time entropy density.

We aim to apply our approach in a next paper to more sophisticated infinite dimensional SDEs called continuous systems, to distinguish them from the lattice case (see the pioneer work from R. Lang [L77]). There, the drift of the $i$ th-coordinate depends on any other coordinate $X_{j}$ according to the values taken by $X_{j}$ and not only to the relative position of the index $j$ with respect to $i$.

The paper is divided into the following sections. Section 2 contains the framework and the main results. In section 3, the proof of the main theorem is given, consisting in the construction of a weak solution of (1) as minimizer of a free energy functional. In section 4, we will point out some interesting structural properties satisfied by this solution.

\section{Framework and main result}

\section{$2.1 \quad$ State spaces}

From now on, without loss of generality, we fix $T=1$, i.e. the time interval is equal to $[0,1]$. So the configuration space of the $\operatorname{SDE}(1)$ is the canonical space $\Omega=\mathcal{C}([0,1], \mathbb{R})^{\mathbb{Z}^{d}}$ endowed with the canonical Borel $\sigma$-field $\mathcal{F}$ generated by the cylinders. The canonical process on $\Omega$ is denoted by $X=\left(X_{i}(t)\right)_{i \in \mathbb{Z}^{d}, t \in[0,1]}$. It generates the canonical filtration which we will use in the sequel.

For any $\omega \in \Omega, i \in \mathbb{Z}^{d}$ and any $t \in[0,1]$, we denote by $\omega^{*}(t)$ the element 
in $\mathbb{R}^{\mathbb{Z}^{d}}$ defined by

$$
\omega_{i}^{*}(t)=: \sup _{0 \leq s \leq t}\left|\omega_{i}(s)\right|
$$

For any $i \in \mathbb{Z}^{d}$, we denote by $\theta_{i}$ the space shift by vector $-i$ which acts on $\mathbb{R}^{\mathbb{Z}^{d}}$ or on $\Omega$. With $\mathcal{P}(E)$ we denote the space of probability measures on any measurable space $(E, \mathcal{E})$. Moreover,

$$
\mathcal{P}_{s}(\Omega):=\left\{P \in \mathcal{P}(\Omega), P \circ \theta_{i}^{-1}=P \quad \forall i \in \mathbb{Z}^{d}\right\}
$$

is the set of probability measures on $\Omega$ which are space-shift invariant.

Similarly,

$$
\mathcal{P}_{s}\left(\mathbb{R}^{\mathbb{Z}^{d}}\right):=\left\{P \in \mathcal{P}\left(\mathbb{R}^{\mathbb{Z}^{d}}\right), P \circ \theta_{i}^{-1}=P \quad \forall i \in \mathbb{Z}^{d}\right\}
$$

In a natural way, we take as reference measure on $\Omega$ the law $\mathbf{W}$ of the noninteracting infinite system corresponding to $\mathbf{b}=0$ with a product measure as initial law, i.e.

$$
\mathbf{W}=\left(\int_{\mathbb{R}} W^{z} m(d z)\right)^{\otimes \mathbb{Z}^{d}} \in \mathcal{P}_{s}(\Omega)
$$

Here $W^{z}$ denotes the Wiener measure on $\mathcal{C}([0,1], \mathbb{R})$ with fixed initial condition $z$ and $m \in \mathcal{P}(\mathbb{R})$ is a given probability measure on $\mathbb{R}$.

For any subset $\Lambda \subset \mathbb{Z}^{d}$ we denote by $X_{\Lambda}=\left(X_{i}\right)_{i \in \Lambda}$ the projection from $\Omega$ on $\mathcal{C}([0,1], \mathbb{R})^{\Lambda}$. We also define the $\sigma$-field

$$
\mathcal{F}_{\Lambda}=\sigma\left(X_{\Lambda}(t), t \in[0,1]\right),
$$

and the projection by $X_{\Lambda}$ of a probability measure $P \in \mathcal{P}(\Omega)$ :

$$
P_{\Lambda}:=P \circ X_{\Lambda}^{-1} \in \mathcal{P}\left(\mathcal{C}([0,1], \mathbb{R})^{\Lambda}\right) .
$$

Similarly, for any $\mu \in \mathcal{P}\left(\mathbb{R}^{\mathbb{Z}^{d}}\right)$, its $\Lambda$-marginal law is denoted by $\mu_{\Lambda} \in \mathcal{P}\left(\mathbb{R}^{\Lambda}\right)$.

\section{$2.2 \quad$ Specific entropy}

For $\mu, \nu$ probability measures on a measurable space $(E, \mathcal{E})$, we denote by $\mathcal{I}(\mu ; \nu)$ their relative entropy defined as usual by: 


$$
\mathcal{I}(\mu ; \nu)=\left\{\begin{array}{ll}
\int_{E} \ln (f) d \mu & \text { if } \mu \ll \nu \text { with density } f \\
+\infty & \text { otherwise }
\end{array} .\right.
$$

When the underlying space has an infinite product structure, i.e. $E=: S^{\mathbb{Z}^{d}}$, one localises the entropy in the following way:

for any subset $\Lambda \subset \mathbb{Z}^{d}$ and $\mu, \nu \in \mathcal{P}\left(S^{\mathbb{Z}^{d}}\right), \mathcal{I}_{\Lambda}(\mu ; \nu):=\mathcal{I}\left(\mu_{\Lambda} ; \nu_{\Lambda}\right)$.

Now, we recall the definition of the specific entropy of a shift invariant probability measure $\mu$ on $S^{\mathbb{Z}^{d}}$ with respect to a reference measure $\sigma \in \mathcal{P}_{s}\left(S^{\mathbb{Z}^{d}}\right)$ :

$$
\mathfrak{I}(\mu):=\lim _{\Lambda \nearrow \mathbb{Z}^{d}} \frac{1}{|\Lambda|} \mathcal{I}_{\Lambda}(\mu ; \sigma),
$$

where the limit above is taken for any increasing sequence $\left(\Lambda_{n}\right)_{n}$ of finite sets converging to $\mathbb{Z}^{d}$ and $|\Lambda|$ denotes the cardinality of $\Lambda$. In the following, we will consider either $S=\mathbb{R}$ and $\sigma=m^{\otimes \mathbb{Z}^{d}}$ or $S=\mathcal{C}([0,1], \mathbb{R})$ and $\sigma=\mathbf{W}$.

The concept of specific entropy appeared first in [RR67] and we advice for instance Chapter 15, [G11] for a general presentation.

\section{$2.3 \quad$ Results}

Let us first define the properties satisfied by the drift and by the initial condition.

- A function $\mathbf{f}$ defined on $\Omega$ is said $(\Delta$-) local if there exists a finite subset $\Delta \subset \mathbb{Z}^{d}$ such that

$$
\mathbf{f}(\omega)=\mathbf{f}\left(\omega_{\Delta}\right)
$$

- A $\Delta$-local adapted drift $\mathbf{b}$ is said uniformly sublinear if there exists $C>0$ such that for all $\omega \in \Omega$ and $t \in[0,1]$,

$$
\mathbf{b}_{t}(\omega)^{2} \leq C\left(1+\sum_{j \in \Delta} \omega_{j}^{*}(t)^{2}\right)
$$

where the paths $\omega_{j}^{*}($.$) were defined by (3).$

A typical example of such a drift, dealt in [RRR10] Equation (20), is $\mathbf{b}_{t}(\omega)=\int_{0}^{t} \alpha\left(s, \omega_{\Delta}(s)\right) d s$, where $\alpha(s, \cdot)$ is a function from $\mathbb{R}^{\Delta}$ to $\mathbb{R}$ with sublinear growth. 
- We denote by

$$
\mathcal{P}_{s, 2}\left(\mathbb{R}^{\mathbb{Z}^{d}}\right):=\mathcal{P}_{s}\left(\mathbb{R}^{\mathbb{Z}^{d}}\right) \cap\left\{\mu: \mathfrak{I}(\mu)<+\infty \text { and } \int x_{0}^{2} \mu(d x)<+\infty\right\}
$$

the set of space-shift invariant probability measure on $\mathbb{R}^{\mathbb{Z}^{d}}$ having a finite specific entropy and a second moment for each coordinate.

Our main result is the following theorem.

Theorem 2.1 Fix an initial probability measure $\mu \in \mathcal{P}_{s, 2}\left(\mathbb{R}^{\mathbb{Z}^{d}}\right)$. Assume that the drift $\mathbf{b}$ is local and uniformly sublinear, i.e. satisfies conditions (6) and (7). Then the infinite-dimensional SDE (1) admits, at least, one shiftinvariant weak solution $P$ with initial marginal law $\mu$. Moreover its specific entropy $\mathfrak{I}(P)$ is finite and $E_{P}\left(\sup _{t \in[0,1]} X_{i}(t)^{2}\right)<+\infty$ for any $i \in \mathbb{Z}^{d}$.

In other words, there exists a probability measure $P \in \mathcal{P}_{s}(\Omega)$ with $\mu$ as marginal at time 0 such that the processes $\left(t \mapsto X_{i}(t)-X_{i}(0)-\right.$ $\left.\int_{0}^{t} \mathbf{b}_{s}\left(\theta_{i} X\right) d s\right)_{i \in \mathbb{Z}^{d}, t \in[0,1]}$ builds a family of $P$-independent Brownian motions. Moreover the finiteness of the specific entropy and the second moment of $\mu$ propagates at the path level.

Remark 2.1 The locality assumption on the drift is relevant: it provides that the approximating dynamics $P^{\xi, \Lambda}$ (defined in Section 3.1) depend on the outside configuration $\xi$ only through its value in a bounded neighbourhood of $\Lambda$. This will allow to recognise the probability kernels $\left(\Pi_{\Lambda}^{H,+}\right)_{\Lambda}$ (see (24)) as local specifications of the probability $\bar{P}$ and therefore leads to the identification of $\bar{P}$ as solution of (1).

We now give a more precise description of the set Sol of weak solutions of the SDE (1) without prescribing the initial condition.

$$
\text { Sol }:=\left\{P \in \mathcal{P}_{s}(\Omega) \text { solution of }(1) \text { with } P \circ X(0)^{-1} \in \mathcal{P}_{s, 2}\left(\mathbb{R}^{\mathbb{Z}^{d}}\right)\right\} .
$$

Theorem 2.2 The set Sol is convex and its extremal points are ergodic solutions. In particular, for any ergodic probability measure $\mu \in \mathcal{P}_{s, 2}\left(\mathbb{R}^{\mathbb{Z}^{d}}\right)$ there exists an ergodic weak solution $P$ of the SDE (1) which admits $\mu$ as marginal law at time 0 . 
More precisely, each probability measure $P$ in Sol admits a unique representation in the following way:

$$
P=\int_{\Theta} \pi(u, .) \vartheta(d u)
$$

where $(\Theta, \mathcal{T}, \vartheta)$ is an auxiliary probability space and $\pi$ is a kernel on $(\Theta, \mathcal{F})$ such that

(i) for each $F \in \mathcal{F}, \pi(., F)$ is $\mathcal{T}$-measurable and

(ii) for each $u \in \Theta, \pi(u,$.$) is an ergodic solution in Sol.$

This theorem is proved in Section 4 which is devoted to the Gibbs structure of the solutions of (1). The proof involves the representation of Gibbs measures by extremal ones.

Let us note that our approach leads to the explicit construction of a particular solution but do not allow to obtain a uniqueness result. For sake of completeness, let us recall a recent result answering this question, obtained via the cluster expansion method, see [RR14] Corollary 2.4. It only concerns the perturbative regime, since the dynamics has to be close to a free dynamics.

Proposition 2.1 Consider the infinite-dimensional SDE (1) with a drift of the form

$$
\left.\mathbf{b}_{t}(\omega):=-\frac{1}{2} \varphi^{\prime}\left(\omega_{0}(t)\right)+\tilde{\mathbf{b}}_{t}\left(\omega_{\Delta}(s), s \in[0, t]\right)\right)
$$

where $\varphi$ is a smooth ultracontractive self-potential (i.e. the semigroup of the associated one-dimensional gradient diffusion maps $L^{2}(m)$ into $\left.L^{\infty}(m)\right)$. Take as initial condition the stationary measure of the free dynamics: $\mu(d x)=$ $\otimes_{i \in \mathbb{Z}^{d}} e^{-\varphi\left(x_{i}\right)} d x_{i}$. If the interaction term $\tilde{\mathbf{b}}$ admits a uniform norm which is sufficiently small, then (1) admits a unique weak solution.

\section{Proof of the main Theorem 2.1}

In this section, we present the proof of Theorem 2.1 divided in several steps. In Section 3.1 we define finite-dimensional approximations of the dynamics (1), and prove some bounds of their second moment in a suitable weighted $\ell^{2}$-space. In section 3.2, we show that a well chosen sequence of approximate solutions with vanishing external configuration is tight for the topology of local convergence on $\Omega$ since their specific entropies are uniformly bounded. 
Then, the identification of any limit point as a Brownian semimartingale with appropriate kernels as local specifications is done in Section 3.3. In Section 3.4, using the previous sections, we prove that any limit point is a zero of the free energy functional, which is computed as the difference between the specific entropy and the specific energy. Thus, in Section 3.5, we complete the proof by identifying the zeros of the free energy as solutions of (1).

\subsection{Finite-dimensional dynamics. Some $\ell^{2}$-bounds.}

For any finite subset $\Lambda \subset \mathbb{Z}^{d}$ and any fixed path $\xi \in \Omega$, we define the $\Lambda$ approximation of the random dynamics (1) with outside frozen configuration $\xi_{\Lambda^{c}}$ and initial fixed condition $\xi(0)$ by means of the SDE

$$
\left\{\begin{aligned}
d X_{i}(t) & =\mathbf{b}_{t}\left(\theta_{i}\left(X_{\Lambda} \xi_{\Lambda^{c}}\right)\right) d t+d B_{i}(t), \quad i \in \Lambda, t \in[0,1] \\
X_{\Lambda}(0) & =\xi_{\Lambda}(0) \\
X_{\Lambda^{c}} & \equiv \xi_{\Lambda^{c}}
\end{aligned}\right.
$$

where the configuration $X_{\Lambda} \xi_{\Lambda^{c}}$ is the concatenation of the configuration $X$ on $\Lambda$ and the configuration $\xi$ outside $\Lambda$. Note that this SDE depends on $\xi_{\Lambda}$ only via its initial value $\xi_{\Lambda}(0)$.

These approximating dynamics will be used in Section 3.2 with $\xi \equiv 0$ and in Section 3.3 for a general outside configuration $\xi$.

Following the framework of [SS80] and [LR85] we introduce the auxiliary Hilbert subspace of $\mathbb{R}^{\mathbb{Z}^{d}}$ defined as weighted $\ell^{2}$-space:

$$
\ell^{2}(\gamma):=\left\{x \in \mathbb{R}^{\mathbb{Z}^{d}},\|x\|_{\gamma}^{2}:=\sum_{i \in \mathbb{Z}^{d}} \gamma_{i} x_{i}^{2}<+\infty\right\}
$$

where $\gamma=\left(\gamma_{i}\right)_{i}$ is the summable sequence $\gamma_{i}:=\frac{1}{(1+|i|)^{d+1}}, i \in \mathbb{Z}^{d}$.

Lemma 3.1 For any $\xi \in \Omega$, the $S D E$ (9) admits a weak solution denoted by $P^{\xi, \Lambda}$. Moreover there exists a constant $K>0$ which does not depend on $\Lambda$ such that

$$
E_{P^{\xi, \Lambda}}\left(\left\|X^{*}(1)\right\|_{\gamma}^{2}\right) \leq K\left(1+\left\|\xi_{\Lambda}(0)\right\|_{\gamma}^{2}+\left\|\xi_{\Lambda^{c}}^{*}(1)\right\|_{\gamma}^{2}\right)
$$

Proof. First, since the growth of $\mathbf{b}$ is sublinear, the growth of the drift of (9) is sublinear too. Therefore $t \mapsto \exp \left(\sum_{i \in \Lambda} \int_{0}^{t} \mathbf{b}_{s}\left(\theta_{i}\left(X_{\Lambda} \xi_{\Lambda^{c}}\right)\right) d B_{i}(s)-\right.$ 
$\left.1 / 2 \int_{0}^{t} \mathbf{b}_{s}^{2}\left(\theta_{i}\left(X_{\Lambda} \xi_{\Lambda^{c}}\right)\right) d s\right)$ is a $\otimes_{i \in \Lambda} W^{\xi_{i}(0)}$-martingale. Applying Girsanov theory, one obtains a weak solution to (9).

To obtain the upper bound (10), we take our inspiration from (4.18) in [SS80] who only treated the particular Markovian case.

First fix $i \in \Lambda$. By Itô formula applied to $X_{i}(t)^{2}$ and (7), one gets for the maximal path

$X_{i}^{*}(t)^{2} \leq X_{i}(0)^{2}+M_{t}^{*}+\int_{0}^{t}\left(X_{i}^{*}(s)^{2}+C\left(1+\sum_{k \in \Lambda} X_{k}^{*}(s)^{2} \mathbb{1}_{k \in i+\Delta}+\sum_{k \in \Lambda^{c}} \xi_{k}^{*}(s)^{2} \mathbb{1}_{k \in i+\Delta}\right)\right) d s+t$

where $M_{t}$ is a martingale with quadratic variation $4 \int_{0}^{t} X_{i}(s)^{2} d s$. Using Doob inequality,

$E\left(M_{t}^{*}\right) \leq \sqrt{E\left(\left(M_{t}^{*}\right)^{2}\right)} \leq 2 \sup _{s \leq t} \sqrt{E\left(M_{s}^{2}\right)} \leq 1+\sup _{s \leq t} E\left(M_{s}^{2}\right) \leq 1+4 \int_{0}^{t} X_{i}^{*}(s)^{2} d s$.

Therefore, denoting by $u_{i}(t)$ the function $t \mapsto E_{P^{\xi, \Lambda}}\left(X_{i}^{*}(t)^{2}\right)$, we obtain

$$
\begin{aligned}
u_{i}(t) \leq & \xi_{i}^{2}(0)+1+4 \int_{0}^{t} u_{i}(s) d s \\
& +\int_{0}^{t}\left(u_{i}(s)+C\left(1+\sum_{k \in \Lambda} u_{k}(s) \mathbb{1}_{k \in i+\Delta}+\sum_{k \in \Lambda^{c}} \xi_{k}^{*}(s)^{2} \mathbb{1}_{k \in i+\Delta}\right)\right) d s+t \\
\leq & \left(\xi_{i}^{2}(0)+C+2+C \sum_{k \in \Lambda^{c}} \xi_{k}^{*}(1)^{2} \mathbb{1}_{k \in i+\Delta}\right)+\sum_{k} Q_{i k} \int_{0}^{t} u_{k}(s) d s
\end{aligned}
$$

where the matrix $Q$ is given by $Q_{i k}=(5+C) \mathbb{1}_{k \in \Lambda \cap i+\Delta}$ for $k \in \mathbb{Z}^{d}$.

For $i \in \Lambda^{c}$, we consider the rough inequality

$$
u_{i}(t) \leq \xi_{i}^{*}(1)^{2}+\sum_{k} Q_{i k} \int_{0}^{t} u_{k}(s) d s .
$$

Remark now that there exists a real number $C^{\prime}>0$ depending only on $\Delta$ but not on $\Lambda$, such that

$$
\forall k \in \mathbb{Z}^{d}, \quad \sum_{i} \gamma_{i} Q_{i k} \leq C^{\prime} \gamma_{k}
$$

Thus, summing over $i$ the inequalities (11) and (12) weighted by $\gamma$, we get

$$
\begin{aligned}
\sum_{i} \gamma_{i} u_{i}(t) \leq & \left\|\xi_{\Lambda}(0)\right\|_{\gamma}^{2}+(C+2) \sum_{i \in \Lambda} \gamma_{i}+\frac{C^{\prime}}{5+C}\left\|\xi_{\Lambda^{+} \backslash \Lambda}^{*}(1)\right\|_{\gamma}^{2}+\left\|\xi_{\Lambda^{c}}^{*}(1)\right\|_{\gamma}^{2} \\
& +C^{\prime} \int_{0}^{t} \sum_{k} \gamma_{k} u_{k}(s) d s,
\end{aligned}
$$


where the term $\left\|\xi_{\Lambda^{c}}^{*}(1)\right\|_{\gamma}^{2}$ could be equal to $+\infty$ if $\xi^{*}(1) \notin \ell^{2}(\gamma)$. This leads by Gronwall's lemma to

$$
\begin{aligned}
E_{P \xi, \Lambda}\left(\left\|X^{*}(t)\right\|_{\gamma}^{2}\right) & \leq\left(\left\|\xi_{\Lambda}(0)\right\|_{\gamma}^{2}+(C+2) \sum_{i \in \Lambda} \gamma_{i}+\frac{C^{\prime}}{5+C}\left\|\xi_{\Lambda^{+} \backslash \Lambda}^{*}(1)\right\|_{\gamma}^{2}+\left\|\xi_{\Lambda^{c}}^{*}(1)\right\|_{\gamma}^{2}\right) e^{C^{\prime} t} \\
& \leq K\left(1+\left\|\xi_{\Lambda}(0)\right\|_{\gamma}^{2}+\left\|\xi_{\Lambda^{c}}^{*}(1)\right\|_{\gamma}^{2}\right)
\end{aligned}
$$

for a constant $K$ which does not depend on $\Lambda$ and is uniformly bounded for $t \in[0,1]$.

In particular, we deduce from the upper bound (10) that, under the assumptions $\xi^{*}(1) \in \ell^{2}(\gamma)$, for any $j \in \mathbb{Z}^{d}$,

$$
E_{P \xi, \Lambda}\left(X_{j}^{*}(1)^{2}\right) \leq \gamma_{j}^{-1} K\left(1+\left\|\xi^{*}(1)\right\|_{\gamma}^{2}\right)<+\infty .
$$

Note that this upper bound is uniform in $\Lambda$ but not in $j$.

\subsection{A tight sequence of approximate solution.}

Take the increasing sequence of finite cubic volume $\Lambda_{n}=\{-n, \ldots, n-1\}^{d} \subset$ $\mathbb{Z}^{d}$. We define the finite-volume approximation of the $\operatorname{SDE}(1)$ on $\Lambda_{n}$ by

$$
\left\{\begin{aligned}
d X_{i}(t) & =\mathbf{b}_{t}\left(\theta_{i}\left(X_{\Lambda_{n}} 0_{\Lambda_{n}^{c}}\right)\right) d t+d B_{i}(t), \quad i \in \Lambda_{n}, t \in[0,1] \\
X_{\Lambda_{n}}(0) & \sim \mu_{\Lambda_{n}} .
\end{aligned}\right.
$$

Its solution $P_{n}$ exists thanks to Lemma 3.1 , and it is given by

$$
P_{n}=\int P_{\Lambda_{n}}^{\xi_{\Lambda_{n} 0_{\Lambda_{n}^{c}}, \Lambda_{n}}} \mu_{\Lambda_{n}}\left(d \xi_{\Lambda_{n}}(0)\right)
$$

With other words, $P_{n}$ is a weak solution of the SDE (9) with vanishing outside configuration and random initial condition following the law $\mu_{\Lambda_{n}}$, restricted to the finite volume $\Lambda_{n}$.

Since $\mu$ admits a finite specific entropy, $\mu_{\Lambda_{n}}$ is absolutely continuous with respect to $m^{\otimes \Lambda_{n}}$ (with density denoted by $f_{\Lambda_{n}}$ ) and by Girsanov Theorem, for any $n$

$$
\begin{aligned}
& \frac{d P_{n}}{d W^{\otimes \Lambda_{n}}}\left(X_{\Lambda_{n}}\right)=f_{\Lambda_{n}}\left(X_{\Lambda_{n}}(0)\right) \exp \left(-H_{\Lambda_{n}}\left(X_{\Lambda_{n}} 0_{\Lambda_{n}^{c}}\right)\right) \\
& \text { where } \\
& \qquad H_{\Lambda}(X)=-\sum_{i \in \Lambda}\left(\int_{0}^{1} \mathbf{b}_{t}\left(\theta_{i} X\right) d X_{i}(t)-\frac{1}{2} \int_{0}^{1} \mathbf{b}_{t}^{2}\left(\theta_{i} X\right) d t\right) .
\end{aligned}
$$


Since we aim at constructing a shift invariant solution of (1), we first introduce a space-periodisation of $P_{n}$. Let $P_{n}^{\text {per }} \in \mathcal{P}(\Omega)$ be the probability measure under which the restrictions of the configurations on disjoint blocks $\left(\left(\theta_{2 k n} X\right)_{\Lambda_{n}}\right)_{k \in \mathbb{Z}^{d}}$ are independent and identically distributed like $P_{n}$. Thus we consider the space-averaged probability measure on $\Omega$

$$
\bar{P}_{n}:=\frac{1}{\left|\Lambda_{n}\right|} \sum_{i \in \Lambda_{n}} P_{n}^{\text {per }} \circ \theta_{i}^{-1} \in \mathcal{P}_{s}(\Omega) .
$$

$\bar{P}_{n}$ is shift invariant by construction. It can be interpreted as the shift invariant extension of the solution of (14) on $\Lambda_{n}$.

We now show that the sequence $\left(\bar{P}_{n}\right)_{n}$ has an accumulation point for the $\mathcal{L}$-topology of local convergence on $\mathcal{P}(\Omega)$. This topology is defined as the coarsest one such that the maps $P \mapsto P(A)$, from $\mathcal{P}(\Omega)$ to $\mathbb{R}$, are continuous for any cylinder $A \in \mathcal{F}$. The key argument is the following tightness criterium based on the specific entropy $\mathfrak{I}$ and proved in [G11], Proposition 15.14.

Proposition 3.1 For any constant $M>0$, the level set

$$
\left\{P \in \mathcal{P}_{s}(\Omega), \mathfrak{I}(P) \leq M\right\}
$$

is sequentially compact for the $\mathcal{L}$-topology.

Therefore, we have to prove such a uniform upper bound for the sequence $\left(\bar{P}_{n}\right)_{n}$.

Proposition 3.2 The specific entropy of the sequence $\left(\bar{P}_{n}\right)_{n}$ is uniformly bounded:

$$
\sup _{n \geq 1} \Im\left(\bar{P}_{n}\right)<+\infty
$$

Proof. First, it is straightforward that

$$
\mathfrak{I}\left(\bar{P}_{n}\right)=\frac{1}{\left|\Lambda_{n}\right|} \mathcal{I}\left(P_{n} ; W^{\otimes \Lambda_{n}}\right) .
$$


(for details, see e.g. the arguments of Proposition 15.52 in [G11]). From (15)

$$
\begin{aligned}
\mathcal{I}\left(P_{n} ; W^{\otimes \Lambda_{n}}\right)= & \int \ln \left(f_{\Lambda_{n}}\right) d \mu_{\Lambda_{n}}-E_{P_{n}}\left(H_{\Lambda_{n}}\left(X_{\Lambda_{n}} 0_{\Lambda_{n}^{c}}\right)\right) \\
= & \mathcal{I}\left(\mu_{\Lambda_{n}} ; m^{\otimes \Lambda_{n}}\right) \\
& +\sum_{i \in \Lambda_{n}} E_{P_{n}}\left(\int_{0}^{1} \mathbf{b}_{t}\left(\theta_{i}\left(X_{\Lambda_{n}} 0_{\Lambda_{n}^{c}}\right)\right)\left(d X_{i}(t)-\mathbf{b}_{t}\left(\theta_{i}\left(X_{\Lambda_{n}} 0_{\Lambda_{n}^{c}}\right)\right) d t\right)\right) \\
& +\frac{1}{2} \sum_{i \in \Lambda_{n}} E_{P_{n}}\left(\int_{0}^{1} \mathbf{b}_{t}^{2}\left(\theta_{i}\left(X_{\Lambda_{n}} 0_{\Lambda_{n}^{c}}\right)\right) d t\right) .
\end{aligned}
$$

Let us first prove that the expectation in the last term of the right hand side of (18) is uniformly bounded (as a function in $i$ and $n$ ). Thanks to the inequalities (7) and (13) and to the stationarity of $\mu$

$$
\begin{aligned}
\|\mathbf{b}\|_{\infty, 2}^{2} & :=\sup _{n} \sup _{i \in \Lambda_{n}} E_{P_{n}}\left(\int_{0}^{1} \mathbf{b}_{t}^{2}\left(\theta_{i}\left(X_{\Lambda_{n}} 0_{\Lambda_{n}^{c}}\right)\right) d t\right) \\
& =\sup _{n} \sup _{i \in \Lambda_{n}} \iint_{0}^{1} \mathbf{b}_{t}^{2}\left(\theta_{i} \omega\right) d t P^{\xi_{\Lambda_{n}} 0_{\Lambda_{n}^{c}}, \Lambda_{n}}(d \omega) \mu_{\Lambda_{n}}\left(d \xi_{\Lambda_{n}}(0)\right) \\
& =\sup _{n} \sup _{i \in \Lambda_{n}} \iint_{0}^{1} \mathbf{b}_{t}^{2}(\omega) d t P^{\theta_{i}\left(\xi_{\Lambda_{n}} 0_{\Lambda_{n}}\right), \theta_{i} \Lambda_{n}}(d \omega) \mu_{\Lambda_{n}}\left(d \xi_{\Lambda_{n}}(0)\right) \\
& \left.\leq C+C K\left(1+\sup _{n} \sup _{i \in \Lambda_{n}} \int_{\mathbb{R}^{\Lambda_{n}}} \| \theta_{i} x_{\Lambda_{n}}\right) \|_{\gamma}^{2} \mu_{\Lambda_{n}}\left(d x_{\Lambda_{n}}\right)\right) \sum_{j \in \Delta} \gamma_{j}^{-1} \\
& \leq C+C K\left(1+\left(\sum_{j \in \mathbb{Z}^{d}} \gamma_{j}\right) \int_{\mathbb{R}^{\mathbb{Z}^{d}}} x_{0}^{2} \mu(d x)\right) \sum_{j \in \Delta} \gamma_{j}^{-1} \\
& <+\infty .
\end{aligned}
$$

Since $P_{n}$ is a weak solution of (14), for each $i \in \Lambda_{n}$, the process $t \mapsto X_{i}(t)-$ $\int_{0}^{t} \mathbf{b}_{s}\left(\theta_{i}\left(X_{\Lambda_{n}} 0_{\Lambda_{n}^{c}}\right)\right) d s$ is a $P_{n}$-Brownian motion. Together with the finiteness of $\|\mathbf{b}\|_{\infty, 2}$, this implies that $t \mapsto \int_{0}^{t} \mathbf{b}_{s}\left(\theta_{i}\left(X_{\Lambda_{n}} 0_{\Lambda_{n}^{c}}\right)\right)\left(d X_{i}(s)-\mathbf{b}_{s}\left(\theta_{i}\left(X_{\Lambda_{n}} 0_{\Lambda_{n}^{c}}\right)\right) d s\right)$ is a $P_{n}$-martingale. So the second term in the right hand side of (18) vanishes. Using the finiteness of the specific entropy of $\mu$, we obtain

$$
\frac{1}{\left|\Lambda_{n}\right|} \mathcal{I}\left(P_{n} ; W^{\otimes \Lambda_{n}}\right) \leq \sup _{n \geq 1} \frac{1}{\left|\Lambda_{n}\right|} \mathcal{I}\left(\mu_{\Lambda_{n}} ; m^{\otimes \Lambda_{n}}\right)+\frac{1}{2}\|\mathbf{b}\|_{\infty, 2}^{2}<+\infty .
$$

With (17), this completes the proof of Proposition 3.2. 
As corollary we get the

Proposition 3.3 There exists a subsequence $\left(\bar{P}_{n_{k}}\right)_{k}$ of the sequence $\left(\bar{P}_{n}\right)_{n}$ which converges for the $\mathcal{L}$-topology to some $\bar{P} \in \mathcal{P}_{s}(\Omega)$.

From now we write for simplicity $\bar{P}=\lim _{n} \bar{P}_{n}$ instead of $\bar{P}=\lim _{k} \bar{P}_{n_{k}}$. The rest of Section 3 is devoted to the analysis of this limit point $\bar{P}$.

\subsection{Structure of the limit point $\bar{P}$}

The class of Brownian semimartingales with bounded specific entropy is closed by $\mathcal{L}$-limits, as we will see in what follows.

\subsection{1 $\quad \bar{P}$ is a Brownian semimartingale}

Recall first the following important structural result for which we give the main lines of the proof.

Lemma 3.2 Let $Q \in \mathcal{P}_{s}(\Omega)$ be a probability measure with finite specific entropy $\Im(Q)$. Then there exists an adapted process $\left(\tilde{\beta}_{t}\right)_{t \in[0,1]}$ in $L^{2}(d t \otimes d Q)$ such that the family of processes

$$
M_{i}(t)=X_{i}(t)-X_{i}(0)-\int_{0}^{t} \tilde{\beta}_{t}\left(\theta_{i} X\right) d s, \quad i \in \mathbb{Z}^{d}, t \in[0,1],
$$

are independent Q-Brownian motions.

\section{Proof.}

First let us notice that the specific entropy $\mathfrak{I}(Q)$ admits the following representation as mean of the relative entropy of a conditional probability:

$$
\mathfrak{I}(Q)=E_{Q}\left(\mathcal{I}_{\{0\}}\left(Q\left(\cdot \mid \mathcal{F}^{-}\right) \mid \mathbf{W}\right)\right),
$$

where $\mathcal{F}^{-}:=\sigma\left(X_{i}, i<0\right)$ (here $<$ denotes the lexicographic order). This result is a version of McMillan theorem, which goes back to the work of Robinson and Ruelle [RR67] and can be proved as in [DP93], Proposition 4.1. Define now $\mathcal{F}^{0}:=\sigma\left(X_{i}, i \neq 0\right)$. Since $\mathcal{F}^{-} \subset \mathcal{F}^{0}$, by Jensen inequality,

$$
E_{Q}\left(\mathcal{I}_{\{0\}}\left(Q\left(\cdot \mid \mathcal{F}^{0}\right) \mid \mathbf{W}\right)\right) \leq E_{Q}\left(\mathcal{I}_{\{0\}}\left(Q\left(\cdot \mid \mathcal{F}^{-}\right) \mid \mathbf{W}\right)\right)<+\infty .
$$


The left hand side in (21), also called local entropy in [FW86], is then finite. Thus, by [FW86] Theorem 2.4, there exists an adapted process $\tilde{\beta}$ in $L^{2}(d t \otimes d Q)$ such that

$$
M_{i}(t)=X_{i}(t)-X_{i}(0)-\int_{0}^{t} \tilde{\beta}_{t}\left(\theta_{i} X\right) d s, \quad i \in \mathbb{Z}^{d}, t \in[0,1]
$$

are independent $Q$-Brownian motions.

Since $\bar{P}$ has a finite specific entropy, applying Lemma 3.2 we deduce that it is a Brownian semimartingale characterized by its drift $\beta$. The proof of Theorem 2.1 is complete provided we show that $\beta_{t}(\omega)=\mathbf{b}_{t}(\omega)$ for $d t \otimes \bar{P}_{-}$ almost all $t$ and $\omega$, and that $\bar{P} \circ X(0)^{-1}$ is equal to $\mu$. These identifications will be completed in Section 3.5. The identification of the drift requires additional tools, which we now develop.

\subsubsection{Local structure of $\bar{P}$}

Define, for $\xi \in \Omega$ and $\Lambda \subset \mathbb{Z}^{d}$, a reference probability kernel on $\Omega$,

$$
\Pi_{\Lambda}^{0}(\xi, d \omega):=\otimes_{i \in \Lambda} W^{\xi_{i}(0)}\left(d \omega_{i}\right) \otimes \delta_{\xi_{\Lambda^{c}}}\left(d \omega_{\Lambda^{c}}\right) .
$$

It corresponds to a Brownian dynamics with fixed initial position inside $\Lambda$ and frozen path outside $\Lambda$. Next we perturb it via the functional defined in (15):

$$
\Pi_{\Lambda}^{H}(\xi, d \omega):=e^{-H_{\Lambda}(\omega)} \Pi_{\Lambda}^{0}(\xi, d \omega) .
$$

Note that $\Pi_{\Lambda}^{H}$ is a probability kernel since $e^{-H_{\Lambda}(\omega)}$ is a $\Pi_{\Lambda}^{0}$-martingale. By Girsanov theory, $\Pi_{\Lambda}^{H}(\xi, d \omega)=P^{\xi, \Lambda}(d \omega)$ that is, it corresponds to the weak solution of (9) on $\Lambda$. We also define a probability kernel with a wider interaction range:

$$
\Pi_{\Lambda}^{H,+}(\xi, d \omega):=\frac{1}{Z_{\Lambda}(\xi)} e^{-H_{\Lambda^{+}}(\omega)} \Pi_{\Lambda}^{0}(\xi, d \omega)
$$

where the set $\Lambda^{+}=\left\{i \in \mathbb{Z}^{d}:(\Delta+i) \cap \Lambda \neq \varnothing\right\}$ is a $\Delta$-enlarged version of the set $\Lambda$. Recall that the finite set $\Delta \subset \mathbb{Z}^{d}$ contains the origin and is the interaction range of $\mathbf{b} . Z_{\Lambda}(\xi)=\int e^{-H_{\Lambda^{+}}(\omega)} \Pi_{\Lambda}^{0}(\xi, d \omega)$ is the normalising constant, usually called partition function in Statistical Mechanics. The family $\left(\Pi_{\Lambda}^{H,+}\right)_{\Lambda}$ will be identified as conditional expectations of $\bar{P}$ with respect to a decreasing sequence of $\sigma$-fields, see Remark 3.1 below and (27). 
Notice that this kernel contains a stochastic integral which is not a priori meaningful. Moreover, it is not trivial why $Z_{\Lambda}(\xi)$ belongs to $] 0,+\infty[$. However, it is the case in our framework, as we show in the next lemma.

Lemma 3.3 The $\operatorname{map} \xi \mapsto \Pi_{\Lambda}^{H,+}(\xi, \cdot)$ is well-defined for $\mathbf{W}$-almost all $\xi$. In particular, it is also $P$-almost surely defined for any probability measure $P$ which is locally absolutely continuous with respect to $\mathbf{W}$.

Proof. The stochastic integrals with respect to $\left(\xi_{i}\right)_{i \in \Lambda^{+} \backslash \Lambda}$ appearing in $\Pi_{\Lambda}^{H,+}(\xi,$.$) are clearly meaningful \mathbf{W}$-almost surely. Moreover, by Girsanov theorem, $E_{\mathbf{W}}\left(Z_{\Lambda}\right)=1$ which ensures that $Z_{\Lambda}$ is $\mathbf{W}$-a.s. finite. Since $H_{\Lambda}$ is $\mathbf{W}$-almost surely finite, $Z_{\Lambda}$ is $\mathbf{W}$-a.s. positive and the lemma is proved.

Remark 3.1 Define, for $\Lambda \subset \mathbb{Z}^{d}$, the $\sigma$-field $\mathcal{G}_{\Lambda}=\sigma\left(X_{\Lambda^{c}}, X(0)\right)$. It builds a decreasing family when $\Lambda$ increases and $\Pi_{\Lambda}^{0}=\mathbf{W}\left(\mid \mathcal{G}_{\Lambda}\right)$ a.s.. Moreover, $\xi \mapsto \Pi_{\Lambda}^{H}(\xi, \cdot)$ is $\mathcal{G}_{\Lambda} \cap \mathcal{F}_{\Lambda^{+}}=\sigma\left(X_{\Lambda^{+} \backslash \Lambda}, X_{\Lambda}(0)\right)$-measurable since $H_{\Lambda}$ is $\mathcal{F}_{\Lambda^{+-}}$ measurable, and $\xi \mapsto \Pi_{\Lambda}^{H,+}(\xi, \cdot)$ is $\partial \mathcal{F}_{\Lambda}$-measurable, where the boundary $\sigma$ field $\partial \mathcal{F}_{\Lambda}$ is defined by $\partial \mathcal{F}_{\Lambda}:=\mathcal{G}_{\Lambda} \cap \mathcal{F}_{\Lambda^{++}} .\left(\Lambda^{++}\right.$is a simplified notation for $\left.\left(\Lambda^{+}\right)^{+}\right)$.

We now present an equilibrium equation - or fixed point property - satisfied by $\bar{P}$ which in fact determines its local specifications, and therefore induces some Gibbsian structure, as we will emphasize in Section 4.

Lemma 3.4 For any finite subset $\Lambda$ of $\mathbb{Z}^{d}$,

$$
\bar{P}(d \omega)=\int_{\Omega} \Pi_{\Lambda}^{H,+}(\xi, d \omega) \bar{P}(d \xi) .
$$

Proof. First, let us note that the right term in (26) is meaningful. Indeed, since the specific entropy of $\bar{P}$ is finite, $\bar{P}$ is locally absolutely continuous with respect to $\mathbf{W}$. Therefore, by Lemma $3.3, \Pi_{\Lambda}^{H,+}(\xi,$.$) is well defined for$ $\bar{P}$-almost all $\xi$.

We have to prove that

$$
\int g(\omega) \bar{P}(d \omega)=\int g(\omega) \Pi_{\Lambda}^{H,+}(\xi, d \omega) \bar{P}(d \xi)
$$

holds for any bounded local measurable function $g\left(g(\omega)=g\left(\omega_{\tilde{\Lambda}}\right)\right.$ for some finite $\left.\tilde{\Lambda} \subset \mathbb{Z}^{d}\right)$. Denote by $\Gamma$ a finite set of $\mathbb{Z}^{d}$ which includes both $\tilde{\Lambda}$ and $\Lambda^{++}$. 
Using standard conditional calculus (see e.g. Lemma 1 and 2 in [DPRZ02]), it is simple to show that for $n$ large enough assuring that $\Lambda_{n} \supset \Gamma$, the probability measure $P_{n}$ satisfies

$$
\int g(\omega) P_{n}(d \omega)=\int g(\omega) \Pi_{\Lambda}^{H,+}(\xi, d \omega) P_{n}(d \xi)
$$

which implies $P_{n}\left(\cdot \mid \mathcal{G}_{\Lambda}\right)=\Pi_{\Lambda}^{H,+} \quad$ a.s..

Noting that $\xi \mapsto \int g(\omega) \Pi_{\Lambda}^{H,+}(\xi, d \omega)$ is local we have

$$
\begin{aligned}
\int g(\omega) \bar{P}(d \omega) & =\lim _{n} \frac{1}{\left|\Lambda_{n}\right|} \sum_{i \in \Lambda_{n}} \int g(\omega) P_{n}^{\text {per }} \circ \theta_{i}^{-1}(d \omega) \\
& =\lim _{n} \frac{1}{\left|\Lambda_{n}\right|} \sum_{i \in \Lambda_{n}, \theta_{i} \Gamma \subset \Lambda_{n}} \int g\left(\theta_{i} \omega\right) P_{n}(d \omega) \\
& =\lim _{n} \frac{1}{\left|\Lambda_{n}\right|} \sum_{i \in \Lambda_{n}, \theta_{i} \Gamma \subset \Lambda_{n}} \int g\left(\theta_{i} \omega\right) \Pi_{\Lambda}^{H,+}(\xi, d \omega) P_{n}(d \xi) \\
& =\lim _{n} \frac{1}{\left|\Lambda_{n}\right|} \sum_{i \in \Lambda_{n}} \int g(\omega) \Pi_{\Lambda}^{H,+}(\xi, d \omega) P_{n}^{\text {per }} \circ \theta_{i}^{-1}(d \xi) \\
& =\int g(\omega) \Pi_{\Lambda}^{H,+}(\xi, d \omega) \bar{P}(d \xi),
\end{aligned}
$$

which is the expected identity.

We interpret the identity (26) as follows: Randomizing under $\bar{P}$ the boundary condition $\xi$ of the kernel $\Pi_{\Lambda}^{H,+}(\xi, \cdot)$ leads back to $\bar{P}$. It implies in particular that

$$
\bar{P}\left(\cdot \mid \mathcal{G}_{\Lambda}\right)=\Pi_{\Lambda}^{H,+} \quad \text { a.s. }
$$

which means that the limiting procedure in $n$ and the conditioning with respect to $\mathcal{G}_{\Lambda}$ are two transformations which commute.

\section{4 $\bar{P}$ minimizes the free energy functional.}

For any probability measure $Q \in \mathcal{P}_{s}(\Omega)$ with finite specific entropy, we define the $Q$-mixtures of the kernels $\Pi_{\Lambda}^{H}$ and $\Pi_{\Lambda}^{H,+}$ by:

$$
\Pi_{\Lambda, Q}^{H}(d \omega)=\int_{\Omega} \Pi_{\Lambda}^{H}(\xi, d \omega) Q(d \xi), \quad \Pi_{\Lambda, Q}^{H,+}(d \omega):=\int_{\Omega} \Pi_{\Lambda}^{H,+}(\xi, d \omega) Q(d \xi) .
$$


With these notations, the equilibrium equation (26) reads as follows:

$\bar{P}$ is a fixed point of the map $Q \mapsto \Pi_{\Lambda, Q}^{H,+}$.

Moreover, if we assume that $\mathbf{b} \in L^{2}(d t \otimes d Q)$, i.e.

$$
E_{Q}\left(\int_{0}^{1} \mathbf{b}_{t}^{2}(X) d t\right)<+\infty
$$

we can define $\mathfrak{I}^{\mathbf{b}}(Q)$, the so-called free energy of $Q$, as the difference between its specific entropy and its specific energy, namely

$$
\mathfrak{I}^{\mathbf{b}}(Q):=\mathfrak{I}(Q)-\mathfrak{I}\left(Q \circ X(0)^{-1}\right)-E_{Q}\left(\int_{0}^{1} \mathbf{b}_{t}(X) d X_{0}(t)-\frac{1}{2} \int_{0}^{1} \mathbf{b}_{t}^{2}(X) d t\right) .
$$

Notice that $\mathfrak{I}^{\mathbf{b}}(Q)$ is well defined although a stochastic integral term occurs. Since $Q$ has a finite specific entropy, by Lemma 3.2, we have that $E_{Q}\left(\int_{0}^{1} \mathbf{b}_{t}(X) d X_{0}(t)\right)$ is nothing but $\left.E_{Q}\left(\int_{0}^{1} \mathbf{b}_{t}(X) \beta_{t}(X)\right) d t\right)$ which is finite because $\beta$ and $\mathbf{b}$ are in $L^{2}(d t \otimes d Q)$.

Note also that, by the convergence of $\left(\bar{P}_{n}\right)_{n}$ to $\bar{P}$ for the local topology, following the computations done in (19), we obtain that

$$
E_{\bar{P}}\left(\left\|X^{*}(1)\right\|_{\gamma}^{2}\right)<+\infty \text { and } E_{\bar{P}}\left(\int_{0}^{1} \mathbf{b}_{t}^{2}(X) d t\right)<+\infty .
$$

Therefore the free energy of $\bar{P}$ is well defined.

In the proposition below we show that $\mathfrak{I}^{\mathbf{b}}$ is a thermodynamical functional, in the sense that it can be also obtained as limit of rescaled finitevolume relative entropies.

Proposition 3.4 Consider $Q \in \mathcal{P}_{s}(\Omega)$ with finite specific entropy and satisfying (28). Then

$$
\mathfrak{I}^{\mathbf{b}}(Q)=\lim _{n} \frac{1}{\left|\Lambda_{n}\right|} \mathcal{I}_{\Lambda_{n}^{+}}\left(Q ; \Pi_{\Lambda_{n}, Q}^{H}\right) .
$$


Proof. By definition of the relative entropy we have

$$
\begin{aligned}
\mathcal{I}_{\Lambda_{n}^{+}}\left(Q ; \Pi_{\Lambda_{n}, Q}^{H}\right)= & E_{Q}\left(\ln \left(\left.\frac{d Q}{d \Pi_{\Lambda_{n}, Q}^{H}}\right|_{\Lambda_{n}^{+}}\right)\right) \\
= & E_{Q}\left(\ln \frac{d Q_{\Lambda_{n}^{+}}}{d W^{\otimes \Lambda_{n}^{+}}}+\ln \frac{d W^{\otimes \Lambda_{n}^{+}}}{d\left(\int \otimes_{i \in \Lambda_{n}} W^{\xi_{i}(0)} Q(d \xi) \otimes W^{\otimes \Lambda_{n}^{+} \backslash \Lambda_{n}}\right)}\right. \\
& \left.+\ln \frac{d\left(\int \otimes_{i \in \Lambda_{n}} W^{\xi_{i}(0)} Q(d \xi) \otimes W^{\otimes \Lambda_{n}^{+} \backslash \Lambda_{n}}\right)}{\left.d \Pi_{\Lambda_{n}, Q}^{0}\right|_{\Lambda_{n}^{+}}}+\left.\ln \frac{d \Pi_{\Lambda_{n}, Q}^{0}}{d \Pi_{\Lambda_{n}, Q}^{H}}\right|_{\Lambda_{n}^{+}}\right) \\
= & \mathcal{I}_{\Lambda_{n}^{+}}(Q ; \mathbf{W})-\mathcal{I}_{\Lambda_{n}}\left(Q \circ X(0)^{-1} ; m^{\otimes \mathbb{Z}^{d}}\right)-\mathcal{I}_{\Lambda_{n}^{+} \backslash \Lambda_{n}}(Q ; \mathbf{W}) \\
& +E_{Q}\left(H_{\Lambda_{n}}\right)
\end{aligned}
$$

The normalised third term of (31) vanishes: by subadditivity of the relative entropy (see Proposition 15.10 in [G11]),

$$
0 \leq \mathcal{I}_{\Lambda_{n}^{+} \backslash \Lambda_{n}}(Q ; \mathbf{W}) \leq \mathcal{I}_{\Lambda_{n}^{+}}(Q ; \mathbf{W})-\mathcal{I}_{\Lambda_{n}}(Q ; \mathbf{W})
$$

and since $\lim _{n}\left|\Lambda_{n}\right| /\left|\Lambda_{n}^{+}\right|=1$ it follows that

$$
\lim _{n} \frac{1}{\left|\Lambda_{n}\right|} \mathcal{I}_{\Lambda_{n}^{+} \backslash \Lambda_{n}}(Q ; \mathbf{W})=0 .
$$

Let us compute the fourth term of (31). By stationarity of $Q$ and by the definition of $H_{\Lambda_{n}}$, we get

$$
E_{Q}\left(H_{\Lambda_{n}}\right)=-\left|\Lambda_{n}\right| E_{Q}\left(\int_{0}^{1} \mathbf{b}_{t}(X) d X_{0}(t)-\frac{1}{2} \int_{0}^{1} \mathbf{b}_{t}^{2}(X) d t\right) .
$$

From (32), (33) inserted in (31) we obtain

$$
\begin{aligned}
& \lim _{n} \frac{1}{\left|\Lambda_{n}\right|} \mathcal{I}_{\Lambda_{n}^{+}}\left(Q ; \Pi_{\Lambda_{n}, Q}^{H}\right)= \\
& \quad \mathfrak{I}(Q)-\mathfrak{I}\left(Q \circ X(0)^{-1}\right)-E_{Q}\left(\int_{0}^{1} \mathbf{b}_{t}(X) d X_{0}(t)-\frac{1}{2} \int_{0}^{1} \mathbf{b}_{t}^{2}(X) d t\right) .
\end{aligned}
$$

Now we are ready for proving that the free energy vanishes under $\bar{P}$.

Proposition 3.5 The probability measure $\bar{P}$ is a zero of the free energy:

$$
\mathfrak{I}^{\mathbf{b}}(\bar{P})=0 .
$$


Proof. The representation (30) implies that the free energy $\mathfrak{I}^{\mathbf{b}}$ is non negative. So the proof of Proposition 3.5 is complete as soon as we can show that $\mathfrak{I}^{\mathbf{b}}(\bar{P}) \leq 0$.

Since $\bar{P}$ is absolutely continuous with respect to $\Pi_{\Lambda_{n}, \bar{P}}^{H}$ with a $\mathcal{F}_{\Lambda_{n}^{+-}}$-measurable density (see Remark 3.1), for any finite set $\Gamma$ containing $\Lambda_{n}^{+}, \mathcal{I}_{\Gamma}\left(\bar{P} ; \Pi_{\Lambda_{n}, \bar{P}}^{H}\right)$ and $\mathcal{I}_{\Lambda_{n}^{+}}\left(\bar{P} ; \Pi_{\Lambda_{n}, \bar{P}}^{H}\right)$ are identical. Taking in particular $\Gamma=\Lambda_{n}^{++}$, one obtains

$$
\mathfrak{I}^{\mathbf{b}}(\bar{P})=\lim _{n} \frac{1}{\left|\Lambda_{n}\right|} \mathcal{I}_{\Lambda_{n}^{++}}\left(\bar{P} ; \Pi_{\Lambda_{n}, \bar{P}}^{H}\right) .
$$

Thanks to Lemma 3.4

$$
\begin{aligned}
\mathcal{I}_{\Lambda_{n}^{++}}\left(\bar{P} ; \Pi_{\Lambda_{n}, \bar{P}}^{H}\right)= & E_{\bar{P}}\left(\left.\ln \frac{d \bar{P}}{d \Pi_{\Lambda_{n}, \bar{P}}^{0}}\right|_{\Lambda_{n}^{++}}+\left.\ln \frac{d \Pi_{\Lambda_{n}, \bar{P}}^{0}}{d \Pi_{\Lambda_{n}, \bar{P}}^{H}}\right|_{\Lambda_{n}^{++}}\right) \\
= & E_{\bar{P}}\left(\left.\ln \frac{d \Pi_{\Lambda_{n}, \bar{P}}^{H,+}}{d \Pi_{\Lambda_{n}, \bar{P}}^{0}}\right|_{\Lambda_{n}^{++}}+\left.\ln \frac{d \Pi_{\Lambda_{n}, \bar{P}}^{0}}{d \Pi_{\Lambda_{n}, \bar{P}}^{H}}\right|_{\Lambda_{n}^{++}}\right) \\
= & -E_{\bar{P}}\left(H_{\Lambda_{n}^{+}}\right)-E_{\bar{P}}\left(\ln \left(Z_{\Lambda_{n}}\right)\right)+E_{\bar{P}}\left(H_{\Lambda_{n}}\right) \\
= & \left|\Lambda_{n}^{+} \backslash \Lambda_{n}\right| E_{\bar{P}}\left(\int_{0}^{1} \mathbf{b}_{t}(X) d X_{0}(t)-\frac{1}{2} \int_{0}^{1} \mathbf{b}_{t}^{2}(X) d t\right) \\
& -E_{\bar{P}}\left(\ln \left(Z_{\Lambda_{n}}\right)\right) .
\end{aligned}
$$

By (34) and (35) the proof of Proposition 3.5 is completed provided that we show that

$$
\lim _{n} \frac{E_{\bar{P}}\left(\ln \left(Z_{\Lambda_{n}}\right)\right)}{\left|\Lambda_{n}\right|} \geq 0 .
$$

Indeed we have

$$
\begin{aligned}
E_{\bar{P}}\left(\ln \left(Z_{\Lambda_{n}}\right)\right) & =\int \ln \left(\int e^{-H_{\Lambda_{n}^{+}}\left(\omega_{\Lambda_{n}} \xi_{\Lambda_{n}^{c}}\right)} \otimes_{i \in \Lambda_{n}} W^{\xi_{i}(0)}(d \omega)\right) \bar{P}(d \xi) \\
& =\int \ln \left(\int e^{\left(H_{\Lambda_{n}}-H_{\Lambda_{n}^{+}}\right)\left(\omega_{\Lambda_{n}} \xi_{\Lambda_{n}^{c}}\right)} e^{-H_{\Lambda_{n}}\left(\omega_{\Lambda_{n}} \xi_{\Lambda_{n}^{c}}\right)} \otimes_{i \in \Lambda_{n}} W^{\xi_{i}(0)}(d \omega)\right) \bar{P}(d \xi) \\
& \geq \int\left(H_{\Lambda_{n}}-H_{\Lambda_{n}^{+}}\right)(\omega) \Pi_{\Lambda_{n}}^{H}(\xi, d \omega) \bar{P}(d \xi) \\
& =\int \sum_{i \in \Lambda_{n}+\backslash \Lambda_{n}} \int_{0}^{1} \mathbf{b}_{t}\left(\theta_{i} \omega\right)\left(d \xi_{i}(t)-\frac{1}{2} \mathbf{b}_{t}\left(\theta_{i} \omega\right) d t\right) \Pi_{\Lambda_{n}}^{H}(\xi, d \omega) \bar{P}(d \xi) .
\end{aligned}
$$


Recall that, for any $\xi$, the probability measure $\Pi_{\Lambda_{n}}^{H}(\xi,$.$) defined in (23)$ corresponds to a weak solution of (9) with fixed initial condition $\xi_{\Lambda}(0)$ and frozen path outside $\xi_{\Lambda^{c}}$. Therefore we deduce from the stationarity of $\bar{P}$ and inequalities (7), (13) and (29)

$$
\begin{aligned}
\sup _{n} \sup _{i \notin \Lambda_{n}} & \int_{\Omega} \int_{0}^{1} \mathbf{b}_{t}^{2}\left(\theta_{i} \omega\right) d t \Pi_{\Lambda_{n}}^{H}(\xi, d \omega) \bar{P}(d \xi) \\
& =\sup _{n} \sup _{i \notin \Lambda_{n}} \int_{\Omega} \int_{0}^{1} \mathbf{b}_{t}^{2}(\omega) d t P^{\xi, \theta_{i} \Lambda_{n}}(d \omega) \bar{P}(d \xi) \\
& \leq \sup _{n} \sup _{i \notin \Lambda_{n}} \int_{\Omega} \int_{0}^{1} C\left(1+\sum_{j \in \Delta} \omega_{j}^{*}(t)^{2}\right) d t P^{\xi, \theta_{i} \Lambda_{n}}(d \omega) \bar{P}(d \xi) \\
& \leq C+C K\left(1+E_{\bar{P}}\left(\left\|X^{*}(1)\right\|_{\gamma}^{2}\right)\right) \sum_{j \in \Delta} \gamma_{j}^{-1} \\
& <+\infty .
\end{aligned}
$$

Thus (36) holds provided that

$$
\inf _{n} \inf _{i \in \Lambda_{n}^{+} \backslash \Lambda_{n}} \int\left(\int_{0}^{1} \mathbf{b}_{t}\left(\theta_{i}(\omega)\right) d \xi_{i}(t)\right) \Pi_{\Lambda_{n}}^{H}(\xi, d \omega) \bar{P}(d \xi)>-\infty
$$

is proved. We use the decomposition of $\xi(t)$ under $\bar{P}$ as a Brownian semimartingale with $\operatorname{drift} \beta \in L^{2}(d t \otimes d \bar{P})$, proved in Section 3.3.1. For any $\Lambda$ and any $i \notin \Lambda$

$$
\begin{aligned}
& \iint_{0}^{1} \mathbf{b}_{t}\left(\theta_{i}(\omega)\right) d \xi_{i}(t) \Pi_{\Lambda}^{H}(\xi, d \omega) \bar{P}(d \xi) \\
= & \iint_{0}^{1} \mathbf{b}_{t}\left(\theta_{i}(\omega)\right)\left(d \xi_{i}(t)-\beta_{t}\left(\theta_{i} \xi\right) d t\right) \Pi_{\Lambda}^{H}(\xi, d \omega) \bar{P}(d \xi) \\
& \quad+\iint_{0}^{1} \mathbf{b}_{t}\left(\theta_{i}(\omega)\right) \beta_{t}\left(\theta_{i} \xi\right) d t \Pi_{\Lambda}^{H}(\xi, d \omega) \bar{P}(d \xi) \\
= & \iint_{0}^{1} \mathbf{b}_{t}\left(\theta_{i}(\omega)\right) \beta_{t}\left(\theta_{i} \xi\right) d t \Pi_{\Lambda}^{H}(\xi, d \omega) \bar{P}(d \xi) \\
\geq & -\left(\iint_{0}^{1} \mathbf{b}_{t}^{2}\left(\theta_{i}(\omega)\right) d t \Pi_{\Lambda}^{H}(\xi, d \omega) \bar{P}(d \xi)\right)^{1 / 2} E_{\bar{P}}\left(\int_{0}^{1} \beta_{t}^{2} d t\right)^{1 / 2}>-\infty
\end{aligned}
$$

uniformly in $\Lambda$ and $i \notin \Lambda$. Inequality (38) and (36) are then proved. 
Therefore the minimum of the free energy is attained on $\bar{P}$ :

$$
\begin{aligned}
\mathfrak{I}^{\mathbf{b}}(\bar{P}) & =0 \\
& =\min \left\{\mathfrak{I}^{\mathbf{b}}(Q), Q \in \mathcal{P}_{s}(\Omega) \text { such that } \Im(Q)<+\infty \text { and } \mathbf{b} \in L^{2}(d t \otimes d Q)\right\},
\end{aligned}
$$

or, with other words, $\bar{P}$ solves a variational principle.

\section{5 $\bar{P}$ is a weak solution of the $\operatorname{SDE}(1)$}

We have to identify the initial marginal law of $\bar{P}$ and its drift.

\subsubsection{The marginal law of $\bar{P}$ at time 0}

Let $g$ be a bounded $\Gamma$-local function on $\mathbb{R}^{\mathbb{Z}^{d}}$, satisfying $g(\omega)=g\left(\omega_{\Gamma}\right)$ for all $\omega \in \mathbb{R}^{\mathbb{Z}^{d}}$. By shift invariance of $\mu$, for all $n \geq 1$ and $i \in \mathbb{Z}^{d}$ such that $\theta_{i}^{-1} \Gamma \subset \Lambda_{n}$

$$
\mu_{\Lambda_{n}} \circ \theta_{i}^{-1}(g)=\mu \circ \theta_{i}^{-1}(g)=\mu(g) .
$$

So

$$
\begin{aligned}
\bar{P} \circ X(0)^{-1}(g) & =\lim _{n \rightarrow \infty} \bar{P}_{n} \circ X(0)^{-1}(g) \\
& =\lim _{n \rightarrow \infty} \frac{1}{\left|\Lambda_{n}\right|} \sum_{i \in \Lambda_{n}} P_{n}^{\text {per }} \circ \theta_{i}^{-1} \circ X(0)^{-1}(g) \\
& =\lim _{n \rightarrow \infty} \frac{1}{\left|\Lambda_{n}\right|} \sum_{i \in \Lambda_{n}, \theta_{i}^{-1} \Gamma \subset \Lambda_{n}} \mu_{\Lambda_{n}} \circ \theta_{i}^{-1}(g)=\mu(g),
\end{aligned}
$$

which proves that $\bar{P} \circ X(0)^{-1}=\mu$.

\subsubsection{Identification of the dynamics under $\bar{P}$}

It only remains to identify the unknown $\operatorname{drift} \beta$ of $\bar{P}$. Let us rewrite the free energy functional of $\bar{P}$ inserting $\beta$ :

$$
\begin{aligned}
\mathfrak{I}^{\mathbf{b}}(\bar{P})= & \mathfrak{I}(\bar{P})-\mathfrak{I}\left(\bar{P} \circ X(0)^{-1}\right)-E_{\bar{P}}\left(\int_{0}^{1} \mathbf{b}_{t}(X)\left(d X_{0}(t)-\beta_{t}(X) d t\right)\right. \\
& \left.+\int_{0}^{1}\left(\beta_{t}(X) \mathbf{b}_{t}(X)-\frac{1}{2} \mathbf{b}_{t}^{2}(X)\right) d t\right) \\
= & \Im(\bar{P})-\mathfrak{I}(\mu)-E_{\bar{P}}\left(\int_{0}^{1}\left(\beta_{t}(X) \mathbf{b}_{t}(X)-\frac{1}{2} \mathbf{b}_{t}^{2}(X)\right) d t\right) .
\end{aligned}
$$


By Proposition 3.5, this quantity vanishes. On the other side, following essentially the proof of Lemma 8 in [DPRZ02] we have

$$
\mathfrak{I}\left(\bar{P} \circ X(0)^{-1}\right)+\frac{1}{2} E_{\bar{P}}\left(\int_{0}^{1} \beta_{t}^{2} d t\right) \leq \mathfrak{I}(\bar{P}) .
$$

Therefore,

$$
\begin{aligned}
0 & \geq E_{\bar{P}}\left(\frac{1}{2} \int_{0}^{1} \beta_{t}^{2}(X) d t-\int_{0}^{1}\left(\beta_{t}(X) \mathbf{b}_{t}(X)+\frac{1}{2} \mathbf{b}_{t}^{2}(X)\right) d t\right) \\
& =\frac{1}{2} E_{\bar{P}}\left(\int_{0}^{1}\left(\beta_{t}(X)-\mathbf{b}_{t}(X)\right)^{2} d t\right)
\end{aligned}
$$

which implies that $\beta_{t}(\omega)=\mathbf{b}_{t}(\omega)$ for $d t \otimes \bar{P}$-almost all $t$ and $\omega$.

It completes the proof that $\bar{P}$ is an infinite-dimensional Brownian diffusion with drift $\mathbf{b}$ and initial law $\mu$.

\section{On the Gibbs property}

In this section, we deal with the Gibbsian structure of solutions of the SDE (1). First recall that the probability measure $\Pi_{\Lambda}^{H,+}(\xi,$.$) is not always well$ defined, as remarked in Section 3.3.2. To circumvent this difficulty take $\Pi_{\Lambda}^{H,+}(\xi,.) \equiv 0$ when the partition function $Z_{\Lambda}(\xi)$ is not finite or when the stochastic integral with respect to $\xi$ in $H_{\Lambda}^{+}$is not defined. In this way the family of kernels $\left(\Pi_{\Lambda}^{H,+}\right)_{\Lambda \in \mathbb{Z}^{d}}$ builds a local specification as introduced by Preston in [Pr76] (2.10)-(2.14), which allows to define associated Gibbs measures.

Definition 4.1 A probability measure $Q$ on $\Omega$ is a Gibbs measure with respect to the specification $\left(\Pi_{\Lambda}^{H,+}\right)_{\Lambda \subset \mathbb{Z}^{d}}$ if, for all finite subset $\Lambda$ of $\mathbb{Z}^{d}$,

$$
Q(d \omega)=\int \Pi_{\Lambda}^{H,+}(\xi, d \omega) Q(d \xi)
$$

Note the similarity with equation (26) where $Q$ appears here in place of $\bar{P}$. It follows that $\bar{P}$ is a Gibbs measure with respect to the specification $\left(\Pi_{\Lambda}^{H,+}\right)_{\Lambda \subset \mathbb{Z}^{d}}$. Actually we obtain a more general result. 
Theorem 4.1 Let $Q$ be a probability measure in $\mathcal{P}_{s}(\Omega)$ with finite specific entropy and a marginal at time 0 belonging to $\mathcal{P}_{s, 2}\left(\mathbb{R}^{\mathbb{Z}^{d}}\right)$. Then $Q$ is a Gibbs measure with respect to the specification $\left(\Pi_{\Lambda}^{H,+}\right)_{\Lambda \subset \mathbb{Z}^{d}}$ if and only if $Q$ is a weak solution of the SDE (1).

\section{Proof.}

"६": it is similar to the proof of Theorem 2.1. Indeed, in Section 3, for proving that $\bar{P}$ is a weak solution of the SDE (1), we only used the fact that $\bar{P}$ satisfies equation (26), its specific entropy is finite and $\overline{\bar{P}} \circ X(0)^{-1} \in \mathcal{P}_{s, 2}\left(\mathbb{R}^{\mathbb{Z}^{d}}\right)$ holds.

" $\Rightarrow "$ : it is straightforward. A similar detailed proof can be found in [DPRZ02], Proposition 1.

To complete this section we present the

\section{Proof of Theorem 2.2.}

Let us recall that a shift invariant probability measure is ergodic if it is trivial on the $\sigma$-field of shift invariant sets.

By previous Theorem 4.1, the set of weak solutions Sol is exactly the set of shift invariant Gibbs measures for which the specific entropy and the second moment of the marginal at time 0 are finite. It is known that the set of stationary Gibbs measure admits a representation by mixing of its extremal points which are ergodic (Theorem 2.2 and 4.1 in [Pr76]). Since the specific entropy functional is affine ([G11], Proposition 15.14), this representation remains valid inside the set of Gibbs measures with finite specific entropy and finite second moment. The first part of the theorem is proved.

Now let $\mu$ be an ergodic probability measure in $\mathcal{P}_{s, 2}\left(\mathbb{R}^{\mathbb{Z}^{d}}\right)$ with finite specific entropy. By Theorem 2.1, there exists a weak solution $P$ of the SDE (1) with initial condition $\mu$. Thanks to the above representation, $P$ is a mixing of ergodic weak solutions of the s.d.e. (1) with respect to various initial laws. But all these initial conditions are necessarily equal to $\mu$, by ergodicity. It means that the mixing is trivial and $P$ is itself ergodic. The second part of the theorem is then proved.

Acknowledgement: This work was supported in part by the Labex CEMPI (ANR-11-LABX-0007-01). We thank two anonymous referees for their fruitful comments which allowed to really improve a first version of this paper. 


\section{References}

[AR91] S. Albeverio and M. Röckner, Stochastic differential equations in infinite dimensions: solutions via Dirichlet forms. Probab. Th. Rel. Fields 89 (1991), 347-386.

[AHMP07] M. Arriojas, Y. Hu, S.-E. Mohammed, S.-E. and G. Pap, A delayed Black and Scholes formula. Stoch. Ana. and Appl. 25 (2007), 471497.

[CRZ96] P. Cattiaux, S. Rølly and H. Zessin, Une approche Gibbsienne des diffusions Browniennes infini-dimensionnelles. Probab. Th. Rel. Fields 104 (1996), 147-179.

[DP93] P. Dai Pra Large deviation and stationary measures for interacting particles systems. Stoch. Proc. Appl. 48 (1993), 9-30.

[DPR06] P. Dai Pra, S. Rølly, An existence result for infinite-dimensional Brownian diffusions with non-regular and non-Markovian drift. Markov Proc. Rel. Fields 10 (2006), 113-136.

[DPRZ02] P. Dai Pra, S. Rølly and H. Zessin, A Gibbs variational principle in space-time for infinite-dimensional diffusions. Probab. Theory Relat. Fields 122 (2002), 289-315.

[DPFPR13] G. Da Prato, F. Flandoli, E. Priola and M. Roeckner, Strong uniqueness for stochastic evolution equations in Hilbert spaces with bounded measurable drift. Annals of Probability 41-5 (2013), 3306-3344.

[DPFRV14] G. Da Prato, F. Flandoli, M. Roeckner and A. Yu. Veretennikov, Strong uniqueness for SDEs in Hilbert spaces with non-regular drift. arXiv:1404.5418v1 (2014).

[DPZ92] G. Da Prato and J. Zabczyk, Stochastic equations in infinite dimensions. Cambridge University Press, 1992.

[D87] J.D. Deuschel, Infinite-dimensional diffusion processes as Gibbs measures on $C[0,1]^{\mathbb{Z}^{d}}$. Probab. Theory Relat. Fields 76 (1987), 325-340.

[D09] D. Dereudre, Existence of Quermass processes for non locally stable interaction and non bounded convex grains. Adv. in Appl. Probab. 41 (2009), 664-681. 
[DDG12] D. Dereudre, R. Drouilhet and H.-O. Georgii, Existence of Gibbsian point processes with geometry-dependent interactions. Probab. Theory Relat. Fields 153 (2012), 643-670

[DR05] D. Dereudre and S. Rœlly, Propagation of Gibbsianness for infinitedimensional gradient Brownian diffusions. J. Stat. Phys. 121 (2005), 511-551.

[DR78] H. Doss and G. Royer, Processus de diffusion associé aux mesures de Gibbs. Z. Wahrsch. Verw. Geb. 46 (1978), 125-158.

[FW86] H. Föllmer and A. Wakolbinger, Time reversal of infinitedimensional diffusions. Stoch. Proc. Appl. 22 (1986), 59-77.

[F82] J. Fritz, Stationary measures of stochastic gradient systems, infinite lattice models. Z. Warsch. Verw. Geb. 59 (1982), 479-490.

[G11] H.-O. Georgii, Gibbs measures and phase transitions. 2nd ed., Berlin, De Gruyter, 2011.

[GH96] H.-O. Georgii and O. Häggström, Phase transition in continuum Potts models. Commun. Math. Phys. 181 (1996), 507-528.

[GST14] H.-O. Georgii, T. Schreiber and C. Thäle, Branching random tessellations with interaction: a thermodynamic view. To appear in Annals of Probability

[L77] R. Lang, Unendlich-dimensionale Wienerprozesse mit Wechselwirkung. Z. Wahrsch. Verw. Geb. 38 (1977), 55-72.

[LR85] G. Leha and G. Ritter, On solutions to stochastic differential equations with discontinuous drift in Hilbert space. Math. Ann. 270 (1985), 109-123.

[M97] X. Mao, Stochastic Differential Equations and Their Applications, Horwood, Chichester (1997)

[Pr76] C. Preston, Random fields, Lecture Notes in Mathematics 714, Springer, Berlin (1976)

[RR67] D.W. Robinson and D. Ruelle, Mean entropy of states in classical statistical mechanics, Comm. Math. Phys. 5 (1967), 288-300. 
[RRR10] F. Redig, S. Rœlly and W. Ruszel, Short-time Gibbsianness for infinite-dimensional diffusions with space-time interaction. J. Stat. Phys.138 (2010), 1124-1144.

[RR14] S. Rœelly and W. Ruszel, Propagation of Gibbsianness for infinitedimensional diffusions with space-time interaction. Markov Proc. Rel. Fields 10 (2014).

[RW87] L.C.G. Rogers and D. Williams, Diffusions, Markov Processes, and Martingales, II. Wiley, New York, 1987.

[R99] G. Royer, Une initiation aux inegalités de Sobolev logarithmiques, Cours Spécialisés, Soc. Math. France, Paris (1999).

[SS80] T. Shiga and A. Shimizu, Infinite dimensional stochastic differential equations and their applications. J. Math. Kyoto Univ. 20-3 (1980), 395416 .

[TP01] L.S. Tsimring, A. Pikovsky, Noise-induced dynamics in bistable systems with delay. Phys. Rev. Lett. 87 (2001), 250602. 